Topiques, études satoriennes

Topoï Studies, Journal of the SATOR

\title{
Le coeur mangé à plusieurs sauces
}

\section{Monique Moser-Verrey}

Volume 5, 2021

Le manger et le boire dans la fiction narrative

URI: https://id.erudit.org/iderudit/1081520ar

DOI: https://doi.org/10.7202/1081520ar

See table of contents

Publisher(s)

SATOR, Société d'Analyse de la Topique Romanesque d'Ancien Régime

ISSN

2369-4831 (digital)

Explore this journal

Cite this article

Moser-Verrey, M. (2021). Le coeur mangé à plusieurs sauces. Topiques, études satoriennes / Topoï Studies, Journal of the SATOR, 5, 1-17.

https://doi.org/10.7202/1081520ar

\section{Article abstract}

The " eaten heart " is a scandalous meal that is to be found in literature from its very start. This story rests on various narrative configurations and topoï rooted in a triangular relationship. The heroine's lover offers her his heart. But her husband turns it into a dish. She actually enjoys this dish but dies as soon as she finds out that she ate her lovers heart. This study follows the variations of the topos both through time and literary genres from the troubadours's courtly poems all the way to Sade's pervers stories. The tragical subject matter also triggered parodies, who either magnified the cannibalism or rejected the appalling meal, preferring a comical resolution. It appears however that from the Middle Ages to the dawn of Romanticism lovers show very little appetite. Their amorous communion truly transcends all greedy misfortunes of this world.
This document is protected by copyright law. Use of the services of Érudit (including reproduction) is subject to its terms and conditions, which can be viewed online.

https://apropos.erudit.org/en/users/policy-on-use/ 


\section{Le cour mangé à plusieurs sauces}

Monique Moser-Verrey

Université de Montréal

La légende du cœur mangé s'est répandue en Occident dès le XII siècle. Elle fait son entrée dans le corpus de la littérature française à travers diverses versions de la biographie d'un troubadour roussillonnais du nom de Guilhem de Cabestany dont on connaît aussi plusieurs chansons. Mais le topos de l'amante qui se régale en toute innocence du cœur de son amant se propage aussi à travers des lais et des romans tout aussi anciens. Que l'on pense au lai de Guiron, un poème breton relayé également au $\mathrm{XII}^{\mathrm{e}}$ siècle dans Le roman de Tristan du poète anglonormand Thomas. Puis, au XIII ${ }^{\mathrm{e}}$ siècle, le Roman du châtelain de Coucy et de la dame de Fayel donne une version légèrement différente du même repas. Par ailleurs, on connaît encore les mélodies de quatorze chansons du Chastelain de Couci qui fut un trouvère picard particulièrement apprécié au tournant du XIII ${ }^{\mathrm{e}}$ siècle pour son grand chant courtois ${ }^{1}$.

Imaginons donc un chevalier doué de toutes les vertus requises pour briller en société. Son milieu admire ses faits d'armes et goûte sa poésie. Il rend hommage à la beauté de l'épouse d'un seigneur de la région. Elle répond à ses avances. Le mari en est instruit et trouve moyen d'éliminer son rival. Mais sa vengeance va plus loin. Il se saisit du cœur de l'amant et le donne à manger à sa femme qui s'en régale. Enfin, il lui révèle ce qu'elle vient de manger. L'épouse meurt sans tarder et le mari sera puni.

Trois éléments de cette histoire intéressent «le manger et le boire dans la fiction narrative ", soit le secret qui entoure la préparation du mets fatal, puis la mise en scène du repas lui-même et enfin la révélation assassine du scandale cannibale. Mon propos sera donc de suivre à travers les siècles cette configuration narrative topique, propre à la légende du cœur mangé, pour en saisir les enjeux et les goûts variés.

\footnotetext{
${ }^{1}$ Hans-Herbert S. Räkel, « Chastelain de Couci », 1994, p. 777. 


\section{De l'érotique courtoise à la perversion sadienne}

Les études médiévales offrent évidemment de nombreuses interprétations intéressantes de la légende du cœur mangé. Une étude s'inscrivant dans l'anthropologie littéraire du Moyen Âge montre, par exemple, que le motif auquel on a affaire dans cette légende s'appuie sur une articulation sémantique profonde selon laquelle le repas cannibale permet « la conjonction sur le mode alimentaire, des identités qui étaient initialement conjointes sur le plan sexuel ${ }^{2}$. » Mais l'ingestion cannibale involontaire du partenaire présuppose une double disjonction, soit le meurtre de l'amant de même que sa transformation en mets. Sur le plan de la fable, il y a donc un double méfait qui constitue la vengeance de l'époux trompé. Mais la sauvagerie de cette vengeance blanchit l'amour extra marital plutôt que de le corriger. Les amants sacrifiés finiront donc par être généralement célébrés. La conclusion de cette étude offre enfin une définition tout à fait acceptable du motif universel actualisé dans la littérature médiévale. C'est la «vengeance cannibale d'une perturbation matrimoniale » dans le cadre d'une idéologie sociale particulière, l'érotique courtoise $^{3}$. Ce cadre dicte le raffinement du repas cannibale. Le cour est cuit et apprêté avant d'être mangé. On peut en conclure que la sauce courtoise se veut appétissante.

Un autre cadre idéologique comme celui de la Révolution, par exemple, qui dénonce « les crimes remarquables de la féodalité ${ }^{4}$, verra la sauvagerie poussée jusqu'à la consommation crue du cœur de l'amant. On trouve cela dans la « Suite de l'histoire de Jérôme » au chapitre XI de $L a$ Nouvelle Justine. Sade écrit: « J'incise enfin le cadavre du jeune homme; j’en extirpe le cœur, pour en barbouiller le visage de ma victime ; je la contrains à en mordre quelques parcelles ; et le fier Jérôme, qui venait de faire la loi à deux individus, la recevait en ce moment de son vit : on ne banda jamais de cette violence-là ${ }^{5}$. » Ici, l'agressivité sexuelle primitive n'est nullement canalisée par des règles. L'interdit nutritionnel est également transgressé ouvertement. Or, les victimes du libertin excessif s'appellent Héloïse et Alberoni, pour ne pas dire Abélard. Cette évocation des célèbres amants, tristement punis par la castration du fauteur, donne bien raison à la lecture

\footnotetext{
2 Jean-Jacques Vicensini, « Figure de l'imaginaire et figure du discours. Le motif du "cœur mangé” dans la narration médiévale », 1991, p. 453.

${ }^{3}$ Ibid. p. 457.

${ }^{4}$ Jacques Antoine Dulaure, Histoire critique de la noblesse, 1790, p. 58 ; et, pour une dénonciation des histoires du cœur mangé datant des $\mathrm{XI}^{\mathrm{e}}$ et XII ${ }^{\mathrm{e}}$ siècles, voir le chapitre 3, p. 55-56.

${ }^{5}$ Marquis de Sade, La nouvelle Justine, ou, Les malheurs de la vertu, O.C., 1986, t. 7, p. 14-15.
} 
anthropologique de la légende du cœur mangé selon laquelle le « sémantisme archétypal » du motif comprend la castration de l'amant, actualisée par la préparation culinaire du cœur extirpé 6 . Voilà, sans doute, le fantasme recouvert par les sauces qui doivent nous intéresser.

\section{De la cardiophagie au cannibalisme}

Ce fantasme est amplement déployé dans une parodie de l'histoire du cœur mangé due au poète Renaud de Beaujeu, qui signe Le lai d'Ignauré, ou, Lai du prisonnier. Ce texte hybride introduit dans le poème courtois dès le tournant du XIII ${ }^{\mathrm{e}}$ siècle une tonalité grivoise empruntée au genre des fabliaux. D'une part, il multiplie les prises du chevalier qui, en véritable Don Juan avant la lettre, s'attache non moins de douze femmes. D'autre part, il donne libre cours à la rage des maris trompés qui démembrent l'Ignauré pour servir à leurs femmes avant tout le phallus dont, à leur idée, elles ont joui goulûment. Le repas cardiophage se transforme alors en une « genitaliaphagie » pour venger l'aspect concret de l'offense faite aux maris. Il semblerait même que dans ce lai le phallus constitue le plat principal tandis que le cœur est servi en garniture ou plat secondaire ${ }^{7}$. Il n'empêche que ce repas est aussi mortifère que tous ceux qui relèvent de la légende du cœur mangé, parce que les amantes adhèrent entièrement à l'idéologie courtoise selon laquelle se vivent leurs aventures. Elles ont forcé l'Ignauré à se prononcer pour une seule d'entre elles, le remettant ainsi sur la bonne voie de l'amour courtois. Puis, elles font d'un seul cœur le deuil de sa mort en jeûnant, après le repas fatidique, jusqu'à ce que mort s'en suive. Cette tragédie exalte la valeur spirituelle de l'union des cœurs et la sauce qui nous intéresse devient métaphysique.

En effet, au Moyen Âge, le cœur est loin d'être uniquement une partie du corps érotisée équivalente au pénis. On observe carrément une fascination pour cet organe mis en écriture et ce dans plus d'un type de discours. Une approche médico-théologique des topoï poétiques évoquant le cœur informe utilement le sens de la légende du cœur mangé. Arracher le cœur du rival équivaut non seulement à supprimer sa vie, mais surtout à « anéantir dans sa source même ce fluide [...] qui, au moins symboliquement, s'apparente à l'âme ${ }^{8} \gg$. L'ennemi est donc éliminé corps et âme par ce geste. En avalant le cœur de l'autre, on s'approprie au contraire son fluide vital et la

\footnotetext{
${ }^{6}$ Vicensini, art. cit., p. 454-455.

${ }^{7}$ Martina Di Febo, « Ignauré », Cahiers de recherches médiévales [En ligne], 5 | 1998, 9 70-71.

${ }^{8}$ Bernard Ribémont, « Le cuer del ventre li as trais », 1991, p. 361.
} 
quintessence de sa force. L'incorporation bénéfique ne nécessite cependant aucune préparation culinaire. Si de plus ce cœur - ou ce corps - est donné, la communion acquiert une valeur spirituelle familière à toute la chrétienté. Mais en dissimulant le cœur de son rival dans un plat cuisiné, le mari jaloux anéantit l'amour vivifiant destiné à sa femme. Ceci se vérifie dans toutes les versions de la légende du cœur mangé qui font mourir l'amant lors d'une croisade. Son cœur, envoyé de l'étranger, est alors un messager d'amour chargé de « réaliser le rêve de la communication des âmes, c'est-à-dire de l'étroite union, cosmique et éternelle, des fluides essentiels. ${ }^{9}$ " Mais l'ingestion involontaire de ce messager précipitera l'union des amants dans la mort.

À l'occasion de sa toute première étude satorienne, Madeleine Jeay élargissait le repérage des messagers d'amour au motif de l'oiseau, et plus particulièrement du rossignol, emblème du poète et de son chant d'amour au Moyen Âge ${ }^{10}$. Que l'on pense au Laostic de Marie de France, mais aussi au surnom de Rossignol donné à l'Ignauré par ses douze amantes. Elle faisait l'hypothèse que les histoires du cœur mangé de l'époque courtoise opéraient dans le même réseau de topoï que la légende ovidienne de Philomèle et Procné reprise à la fin du XII ${ }^{\mathrm{e}}$ siècle dans la Philomena de Chrétien de Troyes. On y retrouve, en effet, le meurtre par vengeance, la mutilation du cadavre, le cannibalisme involontaire et la révélation de ce scandale. Mais les rôles sont inversés, car ce sont les femmes qui se vengent des violences du roi de Thrace en lui faisant manger son propre fils. Puis elles échappent à sa colère, métamorphosées en rossignol et en hirondelle. La proximité des topoï de la violence signalés ici est indéniable, mais l'écart entre les cadres idéologiques de la mythologie grecque, d'une part, et des légendes médiévales, d'autre part, explique pourquoi le cœur arraché et le coffret lui servant de sépulture n'habitent pas la Philomena de Chrétien de Troyes.

\section{Le tombeau violé et le cour avalé}

Tout commence, en ce qui concerne notre propos, par un cœur arraché au corps de l'ennemi, un topos très présent dans l'épopée. Il a bénéficié d'une étude satorienne ${ }^{11}$ où l'on voit que le cœur des traîtres est arraché et détruit par vengeance tandis que celui des héros est conservé dans des tissus et coffrets précieux pour honorer leur fidélité. À l'époque des troubadours, le roi

\footnotetext{
${ }^{9}$ Loc. cit.

${ }^{10}$ Madeleine Jeay, « La cruauté de Philomèle : métamorphoses médiévales du mythe ovidien », 1998, p. 111-119.

${ }^{11}$ Françoise Denis, « Cœur arraché / Cœur mangé : modulations », 1998, p. 95-108.
} 
attribue aussi l'honneur de la sépulture aux amants fidèles plutôt que de soutenir la vengeance du mari offensé. Il entend punir ainsi la dégradation du cœur en mets et la traîtrise du repas cardiophage. À ses yeux le crime est là plus encore que dans le fait que le mari a tué l'amant de sa femme et lui a arraché le cœur. Dans le Roman du châtelain de Coucy et de la dame de Fayel, l'amant devient aussi un héros, car il meurt des suites d'une blessure reçue au champ d'honneur. Dès lors son cœur est excisé, puis enveloppé et déposé dans un coffret pour être apporté à sa maîtresse selon ses ordres. Le crime du mari en est d'autant plus évident, puisqu'il viole cette sépulture afin de préparer le repas cannibale. De plus, il y a un délai entre l'excision du cœur et son arrivée en cuisine. Il faudra soit l'embaumer, soit bien l'épicer...

Cette suite d'événements se trouve illustrée dès le $\mathrm{XV}^{\mathrm{e}}$ siècle dans Le livre des amours du Chastellain de Coucy et de la Dame de Fayel, une version en prose du roman signé Jakemés. L'unique version manuscrite de ce livre se trouve à Lille où elle a été produite en 1467 dans l'atelier du « Maître de Wavrin ${ }^{12}$ ». Cette réécriture du roman en vers réduit l'espace alloué aux prouesses des chevaliers, mais elle développe l'intériorité des personnages et les traite avec empathie $^{13}$. La quarantaine d'aquarelles qui agrémente ce manuscrit, confirme par les sujets des scènes illustrées cette tendance intimiste. Une douzaine d'image seulement montrent les chevaliers à cheval ou réunis entre hommes. On verra dans ce ravissant volume aux reproductions colorées l'arrivée de Coucy ${ }^{14}$, un tournoi ${ }^{15}$, une rencontre avec Fayel ${ }^{16}$, une fête de chevaliers ${ }^{17}$, puis deux images montrant comment Fayel épie Coucy avec son écuyer Gobert ${ }^{18}$, une confrontation à trois où Gobert défend Coucy contre Fayel ${ }^{19}$, le roi Richard sur son trône au moment où Coucy se croise $^{20}$, la bataille contre les Sarrasins ${ }^{21}$ et enfin la victoire de Fayel lorsqu'il force Gobert à lui remettre le précieux coffret contenant le cœur de Coucy avec sa dernière lettre ${ }^{22}$. Toutes les autres

\footnotetext{
${ }^{12}$ Aimé Petit et François Suard (éds.), Le livre des amours du Chastellain de Coucy et de la Dame de Fayel, 1994, p. 7-9.

${ }^{13}$ Annie Combes, « Entre déférence et différence, les ambiguïtés de la mise en prose dans Le livre des amours du Chastellain de Coucy et de la Dame de Fayel », 2015, p. 53-72.

${ }_{14}$ Aimé Petit et François Suard (éds.), Le livre des amours du Chastellain de Coucy et de la Dame de Fayel, 1994, p. 36 et 52 .

${ }^{15}$ Ibid., p. 57 et 63.

${ }^{16}$ Ibid., p. 82.

${ }^{17}$ Ibid., p. 88.

${ }^{18}$ Ibid., p. 128 et 133.

${ }^{19}$ Ibid., p. 136.

${ }^{20}$ Ibid., p. 190.

${ }^{21}$ Ibid., p. 202.

${ }^{22}$ Ibid., p. 214.
} 
images ont pour sujet les épreuves et les joies de l'amour. La dame de Fayel et sa chambrière y jouent les rôles principaux. Elles étudient les plaintes et les lettres de Coucy avant de faciliter les rencontres amoureuses. Le secret de ces amours est percé lors d'un repas de fête par une dame également amoureuse de Coucy. Elle ne mange ni ne boit, mais surprend des regards échangés et un soupir révélateur. C'est elle, la dame de Vermandois, qui causera par jalousie la persécution des amants.

Sur l'illustration de ce repas révélateur ${ }^{23}$ le triangle amoureux est bien dessiné. La traitresse est peinte de dos, l'amant se trouve au centre de l'image et l'amante est aussi retirée que possible à gauche, du côté du cœur évidemment, selon les codes de la peinture de l'époque. On verra que sur toutes ces illustrations, les élans du cœur génèrent des gestes portés vers la gauche, tandis que les gestes agressifs et méchants sont portés vers la droite. La disposition des personnages dans l'image est elle-même chargée de sens. L'épisode du cœur mangé est préparé par deux images présentant la mort de Coucy sur le bateau qui devait le ramener chez lui. Avant de mourir il dicte une lettre adressée à sa bien-aimée pour lui offrir son cœur fidèle ${ }^{24}$. Puis il y a le prélèvement du cœur suivant la mort de Coucy ${ }^{25}$. On voit bien à droite le corps incisé et le cœur tendu vers la gauche où se trouve le coffret. Ce cœur réapparait dans une image montrant la cuisine du château de Fayel. Cette fois-ci il est tendu vers la droite. Le seigneur présente ce morceau de viande à son cuisinier, tandis qu'on plume des poulets encore plus à droite ${ }^{26}$. Le repas lui-même occupe deux images. Méconnaissable, le cœur se trouve maintenant dans l'assiette de la dame qui s'en saisit pour le manger devant son mari ${ }^{27}$. Dans la dernière image, on voit le seigneur de Fayel prendre la parole. Son discours meurtrier est projeté vers la droite où se trouve sa femme. Enfin instruite par son mari qu'elle vient de se régaler du cœur de Coucy, la dame de Fayel s'évanouit à table et mourra peu après ${ }^{28}$. Dans la dernière image de cette série, on voit clairement que ce sont les paroles du seigneur de Fayel qui tuent. Il pérore tandis que sa femme s'effondre.

\footnotetext{
${ }^{23}$ Ibid., p. 116.

${ }^{24}$ Ibid., p. 207.

${ }^{25}$ Ibid., p. 210.

${ }^{26}$ Ibid., p. 217.

${ }^{27}$ Ibid., p. 220.

${ }^{28}$ Ibid., p. 222.
} 


\section{Le repas mortel et la victoire de l'amour}

Après avoir observé les gestes, il va donc falloir s'attarder aux discours échangés d'autant plus qu'ils sont exploités par le théâtre du XVIII ${ }^{\mathrm{e}}$ siècle qui redonnera vie à la légende du cœur mangé sur la scène tragique et même comique. Le roman du XIII siècle, plus près de l'oralité que le livre du $\mathrm{XV}^{\mathrm{e}}$ siècle, donne les répliques de ces scènes en discours direct. Voici donc les paroles qui illustrent ici des topoï concernant le manger et le boire. Après avoir intercepté le coffret qui contient les tresses de sa femme et le cœur embaumé du châtelain, signes d'une union « au ciel, pour la vie éternelle ${ }^{29} »$, Fayel va voir son cuisinier et lui ordonne « de s'appliquer à préparer le coulis de poules et de chapons le plus succulent possible. » Puis il ajoute : «On nous le servira à nous tous et ce sera notre plat principal. Avec ce cœur, tu cuisineras un autre mets que tu serviras exclusivement à ta dame. » Une note de la traductrice précise que « [1] coulis est obtenu après une cuisson lente d'aliments, et notamment de viandes et de légumes qui sont ensuite écrasés ${ }^{30}$. » La tromperie consiste ici à dissimuler le cœur humain dans un coulis identique à celui qui se prépare avec de la volaille. Le topos est celui du cœur caché dans un mets dont la formule topique pourrait se lire ainsi : METS_CACHER_CEUR.

La scène du repas se déroule comme prévu.

Le moment venu, on dressa les tables, tous s'assirent pour le dîner et eurent des hors-d'œuvre à profusion. Ensuite les serviteurs apportèrent le premier coulis, parfaitement réussi. Ils le servirent à tous, tandis que seule la dame reçut le second avec le cœur. Tous mangèrent de bon appétit. La dame fit l'éloge du plat, il lui semblait qu'elle n'en avait jamais goûté de plus savoureux et elle demanda : "Pourquoi notre cuisinier n'en prépare-t-il pas d'autres fois ? Le coût est-il si élevé qu'on ne nous en serve pas plus souvent ? Je le trouve vraiment excellent ${ }^{31}$.

De toute évidence, le mets trompeur enchante l'épouse. Elle mange avec beaucoup de plaisir le cœur de son amant sans le savoir. La formule topique pourrait être dès lors : EPOUSE_APPRÉCIER_CARDIOPHAGIE DÉGUISÉE.

Enfin la parole est à l'époux qui justifie un topos déjà inscrit dans Satorbase sous la formule : CANNIBALISME_PAR_VENGEANCE.

- Dame, ne vous étonnez pas si cette nourriture est excellente, car jamais on ne pourrait retrouver sa pareille, quel que soit l'argent dépensé.

\footnotetext{
${ }^{29}$ Jakemés, Le roman du châtelain de Coucy et de la dame de Fayel (éd. bilingue), 2009, p. 555.

${ }^{30}$ Ibid., p. 573.

${ }^{31}$ Ibid., p. 573-575.
}

Topiques, Études satoriennes, 5, 2020, Le Boire et le manger, https://journals.uvic.ca/index.php/sator/index 
- Quel nom lui donne-t-on, cher seigneur ? Dites-le-moi, s'il vous plaît.

- Dame, calmez-vous. Je vous assure, en toute loyauté, qu'avec ce plat vous avez mangé le cœur de l'homme que vous avez le mieux aimé : c'est le cœur du châtelain de Coucy que l'on vous a donné aujourd'hui. Vous seule en avez été servie, car toute la maisonnée et moi-même, nous avons eu un autre plat, qui lui ressemblait. Vous avez aimé le châtelain de son vivant et cet amour a été ma honte et mon tourment, depuis que je l'ai découvert jusqu'à aujourd'hui. C'est pour assouvir quelque peu ma vengeance que je vous ai fait manger son cœur $^{32}$.

La dame n'en croit rien, puisque Coucy est parti en croisade, mais le coffret, la lettre, le sceau finissent par la convaincre.

Par Dieu, seigneur, j'en suis désespérée, mais puisqu'il en est ainsi, je vous donne l'assurance absolue que jamais plus je ne mangerai, qu'aucune bouchée ne s'ajoutera à une nourriture aussi noble. Mon existence est désormais trop lourde à porter, je ne veux plus vivre. Mort, délivre-moi ${ }^{33}$ !

C'est un suicide que la justice de l'époque interprétera comme un meurtre. L'agonie est longue dans le roman de Jakemés et le narrateur clôt cet épisode en s'exclamant dans la logique du dogme chrétien : « Dieu lui soit miséricordieux ${ }^{34}$ ! »

Le suicide de l'épouse est encore plus dramatique dans les Vies de Cabestany où, poursuivie par son mari, la dame se jette d'un balcon et meurt écrasée au sol. À la Renaissance, lorsque Boccace accueille la légende du cœur mangé dans le Décaméron (39e nouvelle), il préfère cette fin violente même si de nombreux éléments de sa nouvelle coïncident avec les propos du roman de Jakemés. Ce qui ajoute du piquant à la sauce du cœur mangé dans la version de Boccace est une amitié virile entre le mari et l'amant. La trahison de l'ami devient alors la cause de tous les $\operatorname{maux}^{35}$.

La grève de la faim ou le jeûne entrainant la mort de l'épouse accorde plus d'espace à la sensibilité féminine. La dame a le temps de faire l'éloge de son ami et de dire qu'elle meurt «par amour pour lui ${ }^{36} \gg$. Il est sans doute exagéré de penser que la cardiophagie l'a comblée ${ }^{37}$, mais ce jeûne pousse à son extrémité un topos présent à travers tout le roman, soit le manque d'appétit des amoureux. Il faut noter la formule topique AMOUR_COUPER_APPÉTIT.

\footnotetext{
32 Ibid., p. 575.

${ }^{33}$ Ibid., p. 577.

34 Ibid., p. 579.

${ }^{35}$ Mariella Di Maio, Le cœur mangé : Histoire d'un thème littéraire du Moyen Âge au XIX siècle, 2005 , p. 35.

36 Jakemés, Le roman du châtelain de Coucy et de la dame de Fayel (éd. bilingue), 2009, p. 579.

${ }^{37}$ Henri Hauvette, « La 39e nouvelle du Décaméron et la légende du “Cœur mangé” », 1912, p. 199.
}

Topiques, Études satoriennes, 5, 2020, Le Boire et le manger, https://journals.uvic.ca/index.php/sator/index 
Les scènes de repas sont nombreuses dans ce roman situé en Bourgogne. L'abondance des mets et du vin est un thème qui se retrouve sans cesse. À Fayel, où le châtelain est reçu plusieurs fois, les mets sont abondants, mais il est tourmenté par son amour et « ne mange rien ${ }^{38} »$. Lors d'une seconde visite, les hôtes sont « servis de mets à profusion et de bon vin à volonté. [...] Après le dîner, on leur sert, à la satisfaction générale, du bon vin, des pommes et des confitures de gingembre ${ }^{39} »$. Peine perdue, car pour le cœur l'aliment de l'amour passe par la vue et par l'ouie selon l'avis judicieux du narrateur ${ }^{40}$. On pourrait aussi s'étendre sur les repas champêtres offerts après les joutes des chevaliers car ils donnent, au bord de l'Oise et ailleurs, des occasions de rencontres aux amoureux. C'est lors d'une telle fête que la dame de Vermandois, amoureuse du châtelain de Coucy « but et mangea peu ${ }^{41}$ », mais surprit, hélas, le secret de la dame de Fayel.

$\mathrm{Au} \mathrm{XVII}^{\mathrm{e}}$ siècle, ce sont les histoires tragiques qui accueillent le topos du CEEUR_MANGÉ. Il a été inscrit dans Satorbase par notre collègue Eglal Henein il y a de cela plus de vingt ans. Son étude ${ }^{42}$ consacrée au Spectacle III inclus dans Les spectacles d'horreur, publié par Jean-Pierre Camus, Évêque de Belley, en 1630, insiste avec raison sur la richesse des allusions aux mythes et aux textes de l'Antiquité dans cette nouvelle fort dense. Ayant embrassé avec force la Contre-Réforme, Camus diffuse la doctrine post-tridentine en ne craignant pas de choquer, d'interpeler et de faire la morale à ses lecteurs et lectrices. En l'occurrence tous les protagonistes sont dans l'erreur et méritent de recevoir une leçon, à commencer par les parents de l'amoureuse «aveuglés d'ambition et d'avarice ${ }^{43} »$. Tout commence par un topos bien connu, le mariage arrangé qui frustre le désir et l'espoir de la jeune fille et condamne son amoureux à partir à la guerre pour mourir «en la Flandre ${ }^{44}$ ». Le cœur qui en revient avec une lettre adressée à la vertueuse Crisèle deviendra un objet de culte odieux pour le vieux mari qui finit par organiser le viol de la tombe suivi du repas cardiophage. Mis en pâté «avec d'autre viande ${ }^{45}$ » le cour embaumé de Memnon s'attire le qualificatif de chair parfumée ! Avertie que son estomac a « servi de sépulcre au cœur de son $\mathrm{ami}^{46}{ }$ Crisèle est horrifiée. Un parent de Memnon tue le vieux mari

\footnotetext{
${ }^{38}$ Jakemés, Le roman du châtelain de Coucy et de la dame de Fayel, 1986, p.26.

${ }^{39}$ Ibid., p. 30.

${ }^{40}$ Ibid., p. 31-32.

41 Ibid., p. 83.

42 Eglal Henein, « Le cœur mangé... ruminé », 1998, p. 77-88.

${ }^{43}$ Jean-Pierre Camus, Les spectacles d'horreur, 2013, p. 80.

44 Ibid., p. 82.

${ }^{45}$ Ibid., p. 83.

${ }^{46}$ Ibid., p. 84.
} 
jaloux lors d'un duel et l'histoire se termine par un autre topos bien connu, celui de la retraite dans un couvent où Crisèle « acheva sa vie en pleurant ses fautes ${ }^{47}$ ». Virgile est enfin cité pour investir les « premières affections ${ }^{48} »$ de Crisèle d'une dignité classique.

\section{La cardiophagie répudiée par les coeurs sensibles}

Cette même dignité classique est revendiquée pour la légende du cœur mangé au XVIII ${ }^{e}$ siècle lorsque deux tragédies l'illustrent la même année. La mode troubadour est alors censée vivifier la fierté nationale en même temps que la tragédie flirte avec l'horreur ${ }^{49}$. Gabrielle de Vergy de Dormont de Belloy, et Fayel de Baculard d'Arnaud ont paru en 1770. Seule la première de ces tragédies a été représentée, mais pas avant 1777. Sa représentation a enfin suscité une parodie intitulée Gabrielle de Passy signée d'Imbert et d'Ussieux. On peut s'étonner, voire se fâcher ${ }^{50}$ que Gabrielle de Vergy soit confondue dans ces tragédies avec la dame de Fayel, car l'histoire de ses amours trahies, issue de la cour de Bourgogne, ne comprend aucune scène de cardiophagie. $\mathrm{Ce}$ rapprochement n'est cependant pas une invention de nos auteurs. Déjà à la fin du XIII siècle, les chansons du Chastelain de Couci étaient si connues que certaines d'entre elles sont citées dans le roman dont il est le héros, mais aussi dans l'histoire de la Chastelaine de Vergi ${ }^{51}$. Au XVIII siècle, Mlle de Lussan assimile « Gabrielle de Vergi ${ }^{52}$ » à Mme de Fayel dans ses Anecdotes de la cour de Philippe-Auguste. Puis au milieu du siècle, une célèbre romance du duc de Vallière diffusera largement les amours de Raoul de Coucy avec la malheureuse Gabrielle de Vergy :

Tous deux s'aimèrent dès l'enfance

Mais le sort injuste et jaloux

L'avait mise sous la puissance

D'un cruel et barbare époux [...]

Quel est mon crime, disait-elle?

L'innocence devrait toucher.

Je suis et je serai fidèle;

Qu'avez-vous à me reprocher ${ }^{53}$ ?

\footnotetext{
${ }^{47}$ Loc. cit.

${ }^{48}$ Énéide, chant IV, v. 28-29.

${ }^{49}$ Jean-Noël Pascal, Le coun terrible, 2005, p. 1xi-lxii. Je citerai les pièces et les chansons en jeu d'après cette édition critique qui offre un excellent aperçu de la résurgence au théâtre de la légende du « cœur mangé » dans les années 1770.

${ }^{50}$ André Mary (trad.), La chambre des dames : contes et romans du Moyen Âge, 1943, p. 382.

${ }^{51}$ Hans-Herbert S. Räkel, « Castelain de Couci », 1994, p. 776.

${ }_{52}^{52}$ Marguerite de Lussan, Anecdotes de la cour de Philippe-Auguste, 1733, p. 225-250.

${ }^{53}$ Jean-Noël Pascal, Le cour terrible, 2005, p. 230.
} 
Depuis que Jean-Pierre Camus a permis aux parents de l'héroïne d'ajouter leur grain de sel à la sauce du repas cardiophage, ce dernier est offert à une innocente victime dont la vertu est inattaquable. Le contraste avec les fureurs du mari ne saurait être plus marqué. La question est bien sûr de savoir comment introduire l'horreur de ce repas inique sur la scène classique.

Le genre de la tragédie classique l'exige, les protagonistes doivent être présents en un seul lieu. Dans les deux pièces, Coucy arrive donc dès le $\mathrm{III}^{\mathrm{e}}$ acte au château où résident Fayel et Gabrielle. Il y aura duel et les deux rivaux seront mortellement blessés. Tout est en place pour que les morts se multiplient dans la dernière scène. Or, Gabrielle est persuadée que son mari veut l'empoisonner. Le poison est un ingrédient qui règle bien des problèmes sur la scène classique. Il en est donc question, bien qu'aucun poison n'agrémente le plat mortel destiné à Gabrielle ni dans la pièce intitulée Gabrielle de Vergy, ni dans celle intitulée Fayel. En général, on peut affirmer que la pièce de Dormont de Belloy penche vers le pathétique du drame en faisant de Gabrielle le personnage principal. Baculard d'Arnaud choisit, au contraire, de consacrer sa pièce à la fureur vengeresse de Fayel, penchant ainsi d'avantage vers la noirceur et l'énergie de la tragédie romantique. Cette différence d'accent se vérifie à merveille dans les scènes qui présentent le cœur du rival. Ainsi, dans Fayel, le mystère plane autour du festin que le mari veut offrir à sa femme. Mais elle de s'écrier :

\section{GABRIELLE}

Je pénètre Fayel et son affreux silence ; Je ne me trompe point à l'art de sa vengeance ;

Les plus mortels poisons qu'il aura pu choisir, Crois-moi, seront mêlés aux mets qu'on va m'offrir ${ }^{54}$.

Le repas que Gabrielle croit mortel aura lieu hors scène. Elle se réjouit de mourir et ne comprend pas les allusions de Fayel à l'expiation qu'il lui réserve jusqu'au moment où levant un rideau, il lui montre la dépouille de Coucy. Voici enfin la pointe :

\section{FAYEL}

Dans ce sein où mon fer s'est ouvert un passage,

J'ai surpris une lettre, aliment de ma rage :

J'ai su que mon rival, pour prix de ton ardeur,

Voulait qu'après sa mort on te portât son cœur...

\footnotetext{
${ }^{54}$ Ibid., p. 170.
} 
GABRIELLE

Achève... achève... ô Ciel! Quelle terreur soudaine !...

FAYEL

Tu sors de cette table où t'appelait ma haine, Où la vengeance était assise à tes côtés.

\section{GABRIELLE}

Eh bien!

\section{FAYEL}

Parmi les mets que l'on t'a présentés,

Le cour de ton amant... frémis... tu dois m'entendre ${ }^{55}$.

L’impératif de la décence ne permet évidemment pas que la cardiophagie soit nommée ici sur la scène classique, mais ni Gabrielle, ni le public ne peuvent l'ignorer.

Dormont de Belloy renonce, pour sa part totalement, au repas cardiophage de la légende. Le cœur extirpé, livré aux regards de Gabrielle, doit en l'occurrence venger le mari jaloux. Soucieux de ne pas non plus montrer de sang sur la scène, il met entre les mains de Fayel un vase couvert d'une lettre dans lequel se trouve le cœur sanglant de Coucy. Et Fayel d'affirmer :

Quel plaisir pour moi, quand son œil égaré, [celui de Gabrielle]

S'arrêtant sur le cœur qui me fut préféré, Verra, pour châtiment, ce gage de ses crimes !

Je mourrai triomphant près de mes deux victimes ${ }^{56}$.

Il est extraordinaire que par souci de bienséance, un cœur arraché tout dégoulinant de sang fasse son apparition sur la scène classique. Il n'est pas question ici d'ingérer le cœur de Coucy, mais persuadée que ce vase contient du poison, Gabrielle veut néanmoins le porter à ses lèvres :

Allons. - Voici la fin de mon affreux supplice ;

(Elle regarde le vase couvert.)

Et des dons de Fayel le seul que je chérisse :

Mon cœur, vers ce poison, s'élance avec transport ${ }^{57}$.

\footnotetext{
${ }^{55}$ Ibid., p. 182-183.

${ }^{56}$ Ibid., p. 81.

${ }^{57}$ Ibid., p. 82.
} 
C'est en retirant la lettre qui recouvre le vase qu'elle voit enfin « un cour tout sanglant ${ }^{58}$ » et s'effondre.

Ces scènes nous montrent un Fayel singulièrement cruel qui pourrait bien avoir inspiré le marquis de Sade. Son idée d'une mort triomphante «près de [ses] deux victimes » rejoint en quelque sorte l'orgasme puissant ressenti par Jérôme entre ses jeunes victimes Hélö̈se et Alberoni...

De 1777 jusqu'en 1792 la tragédie de Dormont de Belloy fut jouée régulièrement à Paris devant des salles toujours bien remplies et connut en tout 129 représentations. Ce fut un des succès notables du dernier quart du XVIII ${ }^{e}$ siècle ${ }^{59}$. Citée par Dulaure en 1790, cette tragédie n'a sans doute pas non plus échappé à Sade. Il est bien évident que dès lors, la légende du cœur mangé, privée de l'épisode culinaire qui nous intéresse, est centrée sur le cœur extirpé et sanglant que Sade n'hésitera pas à mettre dans la bouche d'Héloïse dans le passage de La nouvelle Justine (1799) cité plus haut. Ainsi la délicatesse de Dormont de Beloy, selon laquelle le cœur mangé est répudié, peut bien avoir amené la perversion sadienne.

Tandis que Gabrielle de Vergy prenait l'affiche au Théâtre-Français le 12 juillet 1777, les Comédiens italiens ordinaires du Roi lançaient le 30 août de la même année Gabrielle de Passy, parodie de Gabrielle de Vergy, en un acte, en prose, et en vaudevilles. Les auteurs de cette farce, Barthélémy d'Imbert et Louis d'Ussieux y présentent six personnages, calqués sur la tragédie de Dormont de Belloy, mais Gabrielle se trouve être l'épouse de Coutel, un anatomiste qui dissèque des animaux : des veaux, des moutons... ${ }^{60}$, autant dire un boucher ! Les cœurs sanglants sont de son domaine, mais il n'y en aura pas sur scène, grâce aux tours que lui jouent Bernic, son assistant et Fric-Frac, l'ami de Coucy renommé en l'occurrence Sans-Souci. Il faut, en effet de l'insouciance pour s'aventurer dans la maison d'un boucher jaloux!

Le cœur est omniprésent dans cette parodie où le mot apparait 59 fois en l'espace de 30 pages $^{61}$ ! On ne se prive pas d'évoquer la boucherie en citant Dormont de Belloy : «Dans mon corps expiré, ta main prendra mon cœur ", puis en vaudeville sur l'air de Tiens, voilà ma pipe:

\footnotetext{
${ }^{58}$ Loc. cit.

${ }^{59}$ Ibid., p. lvii.

${ }^{60}$ Ibid., p. 188.

${ }^{61}$ Ibid., p. 217, note 94.
} 
«Ami, le temps presse; / Fuis ces tristes lieux ; / Porte à ma maîtresse / Ce cœur pour adieux ${ }^{62}$ ». Mais Sans-Souci ne meurt pas et, en revoyant Gabrielle, il cite encore Coucy : « Ce cœur respire, il vit, il brûle encor pour toi ${ }^{63} »$. Coutel en conçoit une rage noire. Il désarme Sans-Souci, ordonne qu'on le saigne ${ }^{64}$... Mais le duel et le meurtre qui s'ensuivent ne sont qu'apparents et Bernic finit par convaincre son maître de ne pas offrir à Gabrielle le cœur de son ami dans un vase, car il lui faudrait ensuite également mourir avec elle :

Oh ! ça, tenez; je vous conseille de finir vos affaires un peu plus gaiement. Cette agonie de Gabrielle ferait un triste tableau. Il sortirait de ce vase des convulsions, des évanouissements, des morts subites : avec tout cela, des sanglots, des cris, des ah!... tous les flacons ouverts... ${ }^{65}$

Ces réactions à la scène du vase contenant le cœur sanglant évoquent le vrai scandale causé au Théâtre-Français lors de la première de Gabrielle de Vergy. La parodie, elle, se termine plutôt par un vaudeville où chaque personnage présente son couplet. Celui que Bernic adresse à Coutel résume parfaitement la double critique adressée à de Belloy : Les intrigues amoureuses n’ont pas leur place dans la tragédie, ni l'horreur du cœur arraché au cadavre d'un héros. Voici donc ce couplet :

Faites, je vous le conseille

Un autre usage du cœur ;

Le mot plaît à notre oreille ;

Mais la chose nous fait peur.

Si faire une tragédie

A pour vous quelque appas :

Ah! du moins, je vous en prie,

Que le cœur n'en soit pas ${ }^{66}$.

Tandis que la parodie médiévale de L'Ignauré amplifiait le repas anthropophage de la légende du cœur mangé en l'offrant à douze dames et en ajoutant au mets vengeur les parties génitales de l'amant, la parodie du XVIII ${ }^{\mathrm{e}}$ siècle, respectueuse des cœurs sensibles du public

\footnotetext{
${ }_{62}^{62}$ Ibid., p. 197.

${ }^{63}$ Ibid., p. 205.

${ }^{64}$ Ibid., p. 208.

${ }^{65}$ Ibid., p. 213-214.

${ }^{66}$ Ibid., p. 217.
} 
féminin, exclut de son propos le cœur arraché et mangé pour ne plus savourer que le mot « cœur » accommodé, en l'occurrence, à une sauce de pure rhétorique burlesque !

\section{Bibliographie}

\section{Euvres et sources}

CAMUS, Jean-Pierre, Les spectacles d'horreur, Paris, Classiques Garnier, 2013, [S. Ferrari (éd.)].

DULAURE, Jacques Antoine, Histoire critique de la noblesse, depuis le commencement de la monarchie jusqu'à nos jours ; où l'on expose ses préjugés, ses brigandages, ses crimes, où l'on prouve qu'elle a été le fléau de la liberté, de la raison, des connoissances humaines, et constamment l'ennemi du peuple et des rois, Paris, Guillot imprimeur-libraire, 1790.

JAKEMÉS, Le livre des amours du Chastellain de Coucy et de la Dame de Fayel, Lille, Presses universitaires de Lille, 1994, [A. Petit et F. Suard (éds.)].

JAKEMÉS, Le roman du châtelain de Coucy et de la dame de Fayel, Troësnes, Corps 9, 1986 [A. Petit et F. Suard (trad.)].

JAKEMÉS, Le roman du châtelain de Coucy et de la dame de Fayel (éd. bilingue), Paris, Honoré Champion, 2009, [C. Gaullier-Bougassas (trad. / ed.)].

MARY, André (trad.), La chambre des dames : contes et romans du Moyen Âge, Paris, Gallimard, 1943.

LUSSAN, Marguerite de, Anecdotes de la cour de Philippe-Auguste, Amsterdam, Jean Pauli et la veuve de P. De Coup, 1733.

RENAUD, de Beaujeu, Le lai de l'Ignauré, ou, Lai du prisonnier, Liège, Vaillant-Carmanne (Textes anciens, Académie royale de langue et de littérature françaises de Belgique), 1938, t. 3, [R. Lejeune-Dehousse (ed.)].

SADE, Donatien Alphonse François marquis de, Euvres compètes du Marquis de Sade, Paris, Pauvert, 1986, 15 t. [A. Le Brun et J.-J. Pauvert (eds.)]. 


\section{Études}

COMBES, Annie, «Entre déférence et différence, les ambiguïtés de la mise en prose dans Le livre des amours du Chastellain de Coucy et de la Dame de Fayel », D. Kullmann et S. Lalonde, Réécritures : Regards nouveaux sur la reprise et le remaniement de textes dans la littérature française et au-delà, du Moyen Âge à la Renaissance, Toronto, Pontifical Institute of Mediaeval Studies, 2015, p. 53-72.

DENIS, Françoise, « Cœur arraché / Cœur mangé : modulations », Études littéraires, vol. 31, no 1, 1998, p. 95-108.

DI FEBO, Martina, «Ignauré. La parodie "dialectique" ou le détournement du symbolisme courtois », Cahiers de recherches médiévales [En ligne], 5 | 1998, mis en ligne le 01 octobre 2007, consulté le 01 octobre 2016. URL : http://crm.revues.org/1482 ; DOI : 10.4000/ crm.1482.

DI MAIO, Mariella, Le coeur mangé : Histoire d'un thème littéraire du Moyen Âge au XIXe siècle, Paris, Presses de l'université Paris-Sorbonne, 2005.

HAUVETTE, Henri, « La 39e nouvelle du Décaméron et la légende du "Cœur mangé” », Romania, t. $41, n^{\circ} 162,1912$, p. $184-205$.

HENEIN, Eglal, « Le cœur mangé... ruminé », Martine DEBAISIEUX et Gabrielle VERDIER (eds.), Violence et fiction jusqu'à la Révolution, Tübingen, Gunter Narr Verlag, 1998, p. 77-88.

JEAY, Madeleine, « La cruauté de Philomèle : métamorphoses médiévales du mythe ovidien », dans Martine DEBAISIEUX et Gabrielle VERDIER (eds.), Violence et fiction jusqu'à la Révolution, Tübingen, Gunter Narr Verlag, 1998, p. 111-119.

PASCAL, Jean-Noël, Le coeur terrible, Perpignan, Presses universitaires de Perpignan, 2005.

RÄKEL, Hans-Herbert S., "Castelain de Couci », MGG. Die Musik in Geschichte und Gegenwart: allgemeine Enzyklopädie der Musik, Personenteil 4, Kassel, Bärenreiter ; Stuttgart, Metzler, 1994, T. 2, P. 4., p. 775-781.

RIBÉMONT, Bernard, « "Le cuer del ventre li as trais” : cœur arraché, cœur mangé, cœur envolé : un regard médico-théologique sur quelques thèmes littéraires ", Le "cuer" au Moyen Âge (réalité et senefiance), Aix-en-Provence, C.U.E.R.M.A. (Senefiance), 1991, no 30, p. 347-361. 
VICENSINI, Jean-Jacques, « Figure de l'imaginaire et figure du discours. Le motif du "cœur mangé” dans la narration médiévale ", Le “cuer” au Moyen Âge (réalité et senefiance), Aixen-Provence, C.U.E.R.M.A. (Senefiance), 1991, no 30, p. 441-459. 